Since it seems that a rapid start of extracorporeal depuration is imperative in determining survival.

1023

\section{PARASITIC INFECTIONS IN CHILDREN UNDER 2 YEARS OLD FROM RURAL AREAS AND THEIR RELATIONSHIP WITH MICRONUTRIENTS DEFICIENCIES}

\author{
C.M. Mihai ${ }^{1}$, A. Balasa ${ }^{1}$, L. Mihai' ${ }^{1}$, V. Stroia ${ }^{1}$, \\ M.R. Stoicescu ${ }^{2}$ \\ ${ }^{1}$ Pediatric, Faculty of Medicine of Constanta, \\ ${ }^{2}$ Microbiology and Immunology, Faculty of \\ Pharmacy of Constanta, Constanta, Romania
}

Background: Micronutrients deficiencies such as iron or zinc deficiency were shown to be associated with parasitic infections.

Objective: Our objective was the detection of parasitic infections in a group of children from rural areas of Constanta County and the correlation with the presence of microcytic anemia and iron and zinc deficiencies.

Material and methods: 455 clinically children were biologically analyzed, to determine their micronutrient status and relationship to parasitic illnesses.

Blood samples were collected to detect hemoglobin, iron and zinc serum levels. Patients were also collected a blood sample for immunologic tests ( detection of toxocara antibodies) and 3 stools samples to detect the presence of intestinal parasites.

Results: $39 \%$ were diagnosed with microcytic hypochromic anemia and $27 \%$ had a latent iron deficiency.

In $28 \%$ of children was revealed a low zinc level. Prevalence of both iron and zinc deficiency was identified in $9.2 \%$ of cases.

Of all children with deficiencies $29 \%$ were diagnosed as infected with Giardia, $12 \%$ with Ascaris lumbricoides and $34 \%$ had anti-Toxocara antibodies .Children with anemia and iron deficiency without anemia were diagnosed with the following parasitic infections: Giardia 14\%, and 1.9\% Ascaris lumbricoides, $7 \%$ had anti-Toxocara antibodies.

In zinc deficient group were found: $34 \%$ infected with Giardia, 14\% with Ascaris lumbricoides., $36 \%$ with anti-Toxocara antibodies.
Conclusions: Iron and zinc deficiencies of micronutrients are major deficiencies in children younger than 2 years. The presence of deficiencies of micronutrients and high prevalence of parasitic diseases in this age group raises issues of implementation of effective prevention programs.

\section{4}

\section{ACIDEMIA WITHOUT ACIDOSIS A TYPICAL PRESENTATION OF PROPIONIC ACIDEMIA}

\author{
A.S. Al-Makadma, A.M. Al-Asmari, I.A. Sandokji \\ Pediatrics, King Fahad Medical City, \\ Riyadh, Saudi Arabia
}

Background: One of the most common recessively inherited organic acidemeias is the Propionic acidosis (PA) which results from propionyle-CoA carboxylase (PCC) enzyme deficiency . Classically this disease presented with high anion gap metabolic acidosis with its clinical consequences. We reviewed the records of 4 patients who were diagnosed as PA with normal $\mathrm{pH}$. All of them were found to have high ammonia level. Diagnosis was confirmed by tendom MS/MS and urine gas chromatography/mass spectrometry (GC/MS). All of them were treated supportively

Methods: We retrospectively reviewed the medical records of 24 patients with a confirmed diagnosis of PA, 4 out of them were excluded because of unclear or/and incomplete documentation.

Results: There were four patients who were diagnosed as PA without Acidosis at the time of presentation. They were 3 females and one male. 3 out of them were the product of consanguineous marriage. All of them presented during the first month of life. The first case was referred only because of the significant family history and decreased activity, the second presented with seizures and anemia, the third had septic like picture and pancytopenia and the fourth one presented with respiratory distress and leukocytosis. All of them had normal $\mathrm{PH}$ and high serum ammonia. Diagnosis was confirmed by using tandem MS/MS and urine gas chromatography/mass spectrometry

Conclusion: The presence of hyperammonemia should increase the suspicion of PA diagnosis even in the presence of normal $\mathrm{pH}$. Diagnosis can be confirmed by tandem MS/MS and urine gas chromatography/mass spectrometry. 